\title{
Branch Correlation Measurements and Analysis in an Indoor Rayleigh Fading Channel for Polarization Diversity Using a Dual Polarized Patch Antenna
}

\author{
M. Hajian, H. Nikookar, Senior Member, IEEE, F. v. der Zwan, and L. P. Ligthart, Fellow, IEEE
}

\begin{abstract}
Spatial diversity techniques are advantageous if the received signals of the diversity branches are sufficiently uncorrelated. Theoretical study using the power unbalance between the two branches employing Laplacian direction of arrival (DOA) distribution is carried out. It is shown that for low value of cross-polarization power ratio (XPR) the correlation has a negligible effect. In order to validate the theoretical results indoor NLOS measurements were carried out. The measurements were done to study the cross-correlation between two received signals of orthogonal polarization using a dual linear polarized patch antenna. The antenna has been designed, built and measured. The results of the channel measurements are given. It is concluded that received signals with orthogonal polarization in indoor Rayleigh fading channels have no significant envelope correlation.
\end{abstract}

Index Terms-Correlation, polarization, Rayleigh channel.

\section{INTRODUCTION}

$\mathbf{T}$ HE constructive or destructive interference of multiple waves in a multipath radio channel causes the signal strength to change rapidly in space, polarization and time domain. This is called small-scale fading. Multipath propagation is caused by the scattering of radio waves and depends strongly on the type of environment. In order to combat the fading it is beneficial to use dual-polarized antennas. This technique is called polarization diversity. Polarization diversity technique uses horizontal and vertical polarization simultaneously to receive the faded radio signal in order to enhance the received signal strength. It is shown that using a horizontal/vertical polarization dual-antenna (first system) provides a diversity gain of 3-4 dB while using $\pm 45^{\circ}$ dual-polarized antennas (second system) can improve the signal strength in the order of 5 to $6 \mathrm{~dB}$ [1]. The first antenna system suffers from power unbalance while the second one is corrupted by the correlation. The relation between these two phenomena is studied in [2]. The performance improvement depends on the cross-correlation of the received signals of the diversity branches. This correlation has been studied theoretically and results were presented in [2]-[4]. In [5], a test system was used to evaluate the polarization diversity performance of different antenna pairs in Rayleigh and Rician environments. In case of dual

Manuscript received February 15, 2005. The review of this letter was arranged by Guest Editors H. Nikookar and R. Prasad.

The authors are with the International Research Centre for Telecommunications-Transmission and Radar (IRCTR), Delft University of Technology, Delft 2628 CD, The Netherlands (e-mail: m.hajian@ewi.tudelft.nl).

Digital Object Identifier 10.1109/LMWC.2005.855390 polarized antennas in addition to the direction of arrival (DOA) distribution, the vertical and horizontal gain pattern of the antenna needs to be taken into the consideration [2], [6] to accurately determine the envelope correlation. In [7], the time of arrival (TOA) model proposed by Saleh and Valenzuela [8] was extended to propose the angular distribution in a non line-of-sight (NLOS) environment.

In this letter, the theoretical and measurement efforts are made to study the branch envelope correlation in polarization domain. Theoretical results have shown that for low value of XPR and not far from sector edge of antenna pattern the envelope correlation is negligible. Indoor measurements were done to validate the theoretical results. In order to measure the envelope correlation coefficient a dual-polarized patch antenna has been designed and measured in the DECT band. The patch was used to measure the indoor channel in the polarization domain. This letter is structured as follows: In Section II the theoretical study of branch correlation in the polarization domain is given. In Section III the measurements procedure is outlined. In Section IV remarks of the results are presented. The letter is concluded in Section V with conclusions.

\section{TheORETICAL StUdy OF CORRELATION}

A system with dual polarized antenna at the receive side and linear polarized at the transmit side is considered. It is shown that this system suffers from power unbalance which directly affects the correlation between the two branches [2]. The correlation $\rho_{c}$ is related to the power unbalanced as

$$
\rho_{c}=\frac{U-1}{U+1} .
$$

Where $U$ is the power unbalanced and is given as

$$
U=\frac{P_{V}}{P_{H}} X P R
$$

where $P_{V}$ is the mean received vertical power when transmitter and receiver are vertically polarized. $P_{H}$ is the mean received horizontal power when transmitter and receiver are horizontally polarized. $X P R$ is the cross-polarization power ratio. It is the ratio of mean received power of vertically polarized receive antenna $\left(P_{V V}\right)$ to the mean received power of horizontally polarized receive antenna $\left(P_{V H}\right)$ when signal is transmitted employing vertically polarized antenna

$$
X P R=\frac{P_{V V}}{P_{V H}} .
$$




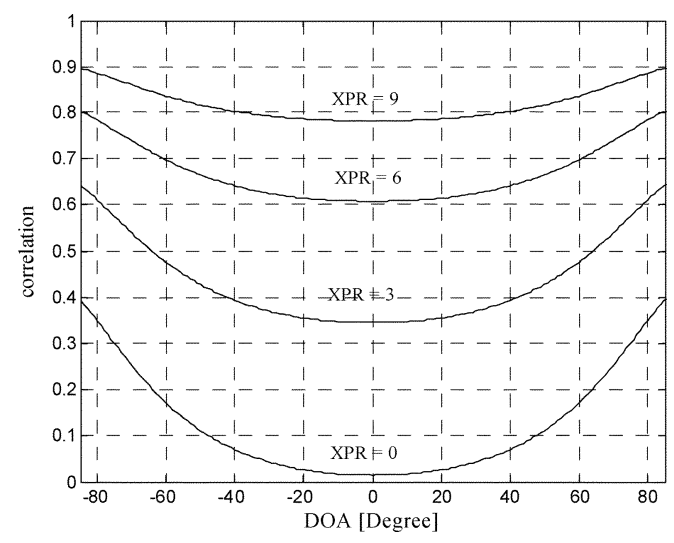

Fig. 1. Power correlation as function of DOA for different values of $X P R$ (in dB). $\sigma=24^{\circ}$.

The vertical and horizontal mean power given in (2) can be studied using the model proposed by [2] and [6]

$$
P_{Y}(\Phi)=P_{Y}^{0} \int_{-\pi}^{\pi} p_{Y}(\Phi, \phi) G_{Y}(\phi) d \phi
$$

where subscript $Y \in\{V, H\}$ corresponds to variable with respect to $Y$ polarization. $G_{Y}(\phi)$ is the antenna power gain pattern in the azimuth plane, $p_{Y}(\Phi, \phi)$ is the distribution of the DOA. $P_{Y}^{0}$ is the mean signal power when $G_{Y}=1$. Parameter $\Phi$ is the DOA of multipath signal, and $\phi$ is the azimuth angle. In order to use (4) the distribution of DOA is needed. It is assumed that the distribution is same for the vertically and horizontally polarized signals. The Laplacian distribution with zero mean proposed in [7] is used and is given as

$$
p(\Phi)=\frac{1}{\sqrt{2} \sigma} e^{-\left|\frac{\sqrt{2} \Phi}{\sigma}\right|}
$$

where $\sigma$ is the deviation of the power azimuth distribution. Fig. 1 shows the power correlation as function of the DOA. The lowest correlation is observed when XPR is low and the DOA is not far from edge. It is shown that the power- and envelope correlation have the same behavior [2].

\section{MEASUREMENTS}

In order to study the correlation, measurements are done in indoor NLOS environments, which can be modeled by Rayleigh propagation. A continuous wave (CW) in the DECT frequency band via a linear-polarized patch antenna is transmitted. A dual-polarized patch antenna is used as receiver antenna, mounted on a mobile wooden stand and can be moved along a random path. The received signals are down converted, sampled and stored for offline post-processing. The paths and directions were chosen randomly and long enough to average over a large number of specific propagation situations. The measurement set up was not suitable to determine DOA of multipath components. For clarity a block diagram of the set up is shown in Fig. 2 [9], [10]. The CW is produced by a "Marconi Signal Generator 2024" at a frequency of $1.89 \mathrm{GHz}$ and at the maximum output power of $+13 \mathrm{dBm}$. The signal is split into a transmit signal and a local oscillator (LO) signal for down conversion of the received signals. An amplifier with a gain of $20 \mathrm{~dB}$ is placed in front of the transmitting patch antenna. The patch was mounted on top of a

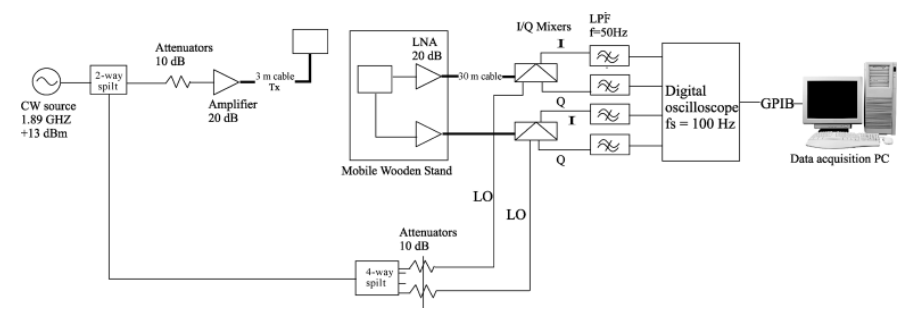

Fig. 2. Block diagram of the hardware setup.

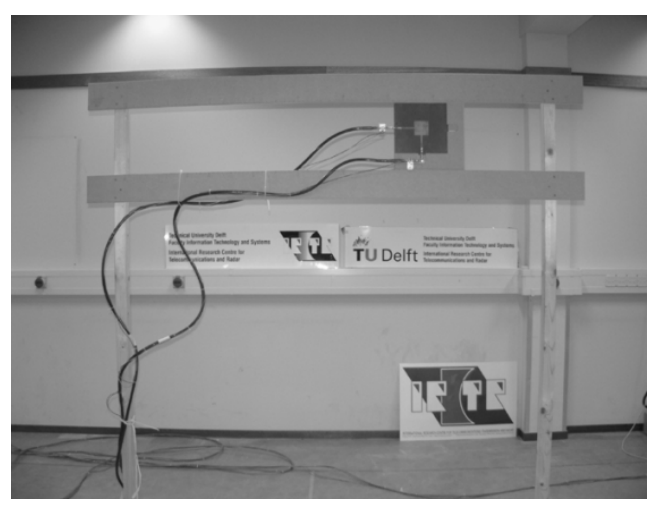

Fig. 3. Mobile wooden stand with the LNAs and receive antenna.

synthetic stand of $1.70 \mathrm{~m}$ height transmitting at vertical polarization. Including the losses in the splitter and the attenuator, which was needed to protect the amplifier, we had about $20 \mathrm{dBm}$ signal power at the input of the transmitting patch. Measurements showed that the average signal-to-noise ratio (SNR) along the measured path was at least $40 \mathrm{~dB}$, which allows accurate measurements with deep fades caused by small-scale fading. The signal was received by dual polarized patch antenna, which was mounted on a mobile wooden stand at $1.70 \mathrm{~m}$ height. Low noise amplifiers (LNA) with a gain of $20 \mathrm{~dB}$ were placed directly after the antennas. Two long radio frequency (RF) cables of $30 \mathrm{~m}$ were used to provide enough mobility for the stand. The envelopes of both received signals were acquired by down-converting the signals in two IQ-mixers. The input level at the mixers was maximum $-20 \mathrm{dBm}$. The LO signal was split and attenuated, which resulted in a $-10 \mathrm{dBm}$ signal strength at the LO input of the mixers. In order to improve the SNR, the two in-phase (I) and the two quadrature-phase (Q) signals were filtered with low pass filters before they were sampled with a "Tektronix Digital Scope TDS 784A." The samples were read from the scope by using the GPIB interface, and were finally stored on a computer for offline post-processing.

Generally, in an indoor environment deep fades of a small-scale fading signal can be found over a distance of about a wavelength. At frequency of $1.89 \mathrm{GHz}$ the wavelength is $15.8 \mathrm{~cm}$. Since the stand was moved with a speed of $0.5 \mathrm{~m} / \mathrm{s}$, which corresponds to about three wavelengths per second, a sampling frequency of $100 \mathrm{~Hz}$ was enough to accurately record the fading signals. The $-3 \mathrm{~dB}$ point of the low pass filters was set to $50 \mathrm{~Hz} .100000$ samples were needed to attain a repeatable correlation coefficient with an accuracy of 0.01 . Sampling at $100 \mathrm{~Hz}$, the measurement time was $1000 \mathrm{~s}$, which corresponds to $16 \mathrm{~min}$ and $40 \mathrm{~s}$. Fig. 3 shows the mobile wooden stand including the LNAs and receive antenna. 


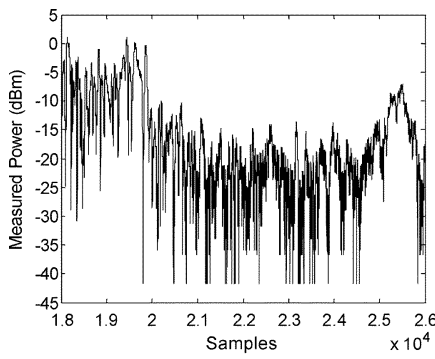

(a)

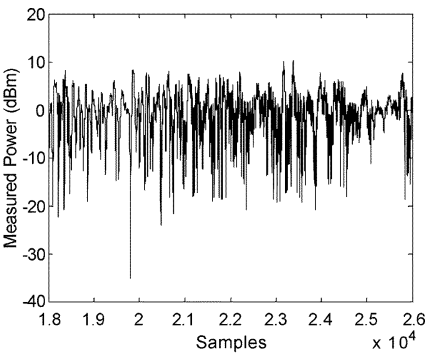

(b)
Fig. 4. (a) Example of measured signal strength of one of the receive branches. (b) De-meaned signal.

A linear- and dual polarized patch antenna is used as transmit and receive antenna, respectively [10]. The antennas are tuned to $1.89 \mathrm{GHz}$. An input reflection and an isolation of $-35 \mathrm{~dB}$ is measured. The measurements were done in IRCTR anechoic room. A moderate gain of 6-7 dBi is measured. Both ports resulted into similar behavior which is a necessity for allowing accurate channel measurements in the polarization domain. The measurements were carried out in an office area. The hall had enough space to move around the receiving stand using different routes. The transmitter was located in a large room. In this way we created a NLOS situation, where the transmitted signal arrives at the receiving antenna via multiple paths scattered by surrounding walls and objects. These paths, all arriving with their own, independent phases, sum up at the antenna and result in a Rayleigh fading channel. By following long and random measurement routes in different directions we averaged over different specific propagation situations. In this way the measured correlation coefficient should lead to a general coefficient, valid for indoor environments. The raw data acquired from the digital oscilloscope contains the sampled signal amplitudes of the I and Q channels of the two branches, i.e., we obtained a matrix of four channels by 100000 samples. The following steps were performed for data processing.

1) The dc offsets removed from all four I and Q channels by subtracting the corresponding means.

2) Long-term path loss and shadowing causes large-scale fading and is super-imposed on small-scale fading. This large-scale fading is removed by normalizing the two signal envelopes with a moving average over six wavelengths, which corresponds in our measurement with an averaging window over 200 samples. This, so called, local "de-meaning" was implemented as follows.

a) The mean of the absolute amplitude was taken for each window.

b) The means of all windows were interpolated using the cubic spline method.

c) The signal envelopes were normalized by dividing them by the corresponding interpolated means.

The effect of "de-meaning" is shown in Fig. 4(a) and (b).

3) The envelope correlation coefficient was calculated as

$$
\rho_{e n v}=\frac{\sum_{i=1}^{N}\left[\left(r_{i 1}-\bar{r}_{1}\right)\right]\left[\left(r_{i 2}-\bar{r}_{2}\right)\right]}{\sqrt{\sum_{i=1}^{N}\left[\left(r_{i 1}-\bar{r}_{1}\right)\right]^{2}\left[\left(r_{i 2}-\bar{r}_{2}\right)\right]^{2}}} .
$$

Where $r_{i 1}$ and $r_{i 2}$ are the signal envelopes. $i$ refers to the sample number, The measurement results have shown that the received signals at two polarizations have no significant envelope correlation in an indoor Rayleigh fading channel. The value of envelope correlation was lower than 0.1 . In this letter only the envelope correlation is considered. However consideration of complex cross-correlation which is specified by the cross-covariance [11] between the received complex signals might be useful to be further investigated.

\section{REMARKS}

There is a good agreement between the theoretical and measurement results. Examining Fig. 1 the low value of correlation corresponds to $-50^{\circ} \leq \mathrm{DOA} \leq 50^{\circ}$ and a low value of XPR. Our measurements results show that the XPR had very low value due to the same gain pattern. Since we have not measured DOA then the mean value of DOA should be in the given range.

\section{CONCLUSION}

The envelope correlation coefficients between horizontal and vertical polarization have been determined theoretically and measured using a dual polarized patch antenna. We conclude that the received signals of the dual polarized patch antenna in an indoor NLOS propagation scenario show no significant envelope correlation.

\section{REFERENCES}

[1] D. Emmer, E. Humburg, P. Weber, and M. Weckerle, "Measurements of base station two branch space and polarization diversity reception and a comparison of the diversity gain based on the CDF of signal level and simulations of BER in a GSM system," in Proc. 48th IEEE VTC'98, vol. 1, New York, NY, 1998, pp. 5-10.

[2] J. Hämäläinen, R. Eichman, J. Hulkkonen, T. Kähkönen, T. Korpi, and M. Säily, "On the performance of GSM/EDGE transmit diversity schemes when employing dual-polarized antennas," in Proc. 57th IEEE Semiannual VTC'03, vol. 4, 2003, pp. 2418-2422.

[3] R. G. Vaughan, "Polarization diversity in mobile communications," IEEE Trans. Veh. Technol., vol. 39, no. 3, pp. 177-186, Aug. 1990.

[4] K. Cho, T. Hori, and K. Kagoshima, "Effectiveness of four-branch height and polarization diversity configuration for street microcell," IEEE Trans. Antennas Propagat., vol. 46, no. 6, pp. 776-781, Jun. 1998.

[5] R. M. Narayanan, K. Atanassov, V. Stoiljkovic, and G. R. Kadambi, "Polarization diversity measurements and analysis for antenna configurations at $1800 \mathrm{MHz}$," IEEE Trans. Antennas Propagat., vol. 52, no. 7, pp. 1795-1810, Jul. 2004.

[6] T. Taga, "Analysis of mean effective gain of mobile antennas in land mobile radio environment," IEEE Trans. Veh. Technol., vol. 39, no. 2, pp. 117-131, May 1990.

[7] Q. H. Spencer, B. D. Jeffs, M. A. Jensen, and A. L. Swindlehurst, "Modeling the statistical time and angle of arrival characteristics of an indoor multipath channel," IEEE J. Selected Areas Commun., vol. 18, no. 3, pp. 347-360, Mar. 2000.

[8] A. A. M. Saleh and R. A. Valenzuela, "A statistical model for indoor multipath propagation," IEEE J. Select. Areas Commun., vol. SAC-5, pp. 128-137, Feb. 1987.

[9] M. Rijken, M. Hajian, F. van der Zwan, and L. P. Ligthart, "Branch correlation measurements in an indoor Rayleigh fading channel using closely spaced monopoles," in Proc. ECWT'03, Munich, Germany, Oct. 6-10, 2003.

[10] M. Hajian, F. van der Zwan, and L. P. Ligthart, "Branch correlation measurements in an indoor Rayleigh fading channel for polarization diversity using a dual polarized patch antenna," in Proc. ECWT'04, Amsterdam, The Netherlands, Oct. 11-15, 2004.

[11] J. Wallace, H. Ozcelik, M. Herdin, E. Bonek, and M. Jensen, "Power and complex envelope correlation for modeling measured indoor MIMO channels: a beamforming evaluation," in Proc. 58th IEEE VTC'03, vol 1, Oct. 6-9, 2003, pp. 363-367. 